\title{
ROLE OF MOLECULAR DIAGNOSTIC TEST IN DIAGNOSING PULMONARY AND EXTRAPULMONARY TUBERCULOSIS
}

\author{
Sheela Mathew ${ }^{1}$, Manjunath M. Pradhani², Beena Philomina ${ }^{3}$ \\ ${ }^{1}$ Additional Professor, Department of Infectious Diseases, Government Medical College Hospital, Kozhikode, Kerala, India. \\ ${ }^{2}$ Senior Resident, Department of Medicine, Government Medical College Hospital, Kozhikode, Kerala, India. \\ 3Professor, Department of Microbiology, Government Medical College, Kozhikode, Kerala, India.
}

\begin{abstract}
BACKGROUND
ABSTRACT

Mycobacterium tuberculosis remains one of the most significant causes of death from an infectious agent. The rapid diagnosis of tuberculosis and detection of Rifampicin resistance are essential for early disease management. The Gene-Xpert MTB/RIF assay is novel integrated diagnostic device for the diagnosis of tuberculosis and rapid detection of RIF resistance in clinical specimens. This study is to assess the role of molecular diagnostic test, the Gene-Xpert in diagnosing Tuberculosis.
\end{abstract}

Aims and Objectives-

1. Application of newer molecular diagnostic test, Gene-Xpert for the diagnosis of tuberculosis in suspected TB patients.

2. To find out sensitivity and specificity of Gene-Xpert.

3. To find out the incidence of drug resistance in newly detected TB with the help of Gene-Xpert.

\section{MATERIALS AND METHODS}

This is a prospective observational study which was conducted in Government Medical College, Kozhikode. A total of 100 patients were included who met the inclusion criteria. Patients were diagnosed to have TB by AFB culture, smear positivity and also clinically. These diagnosed TB patients were grouped into culture positive and negative, smear positive and negative and GeneXpert positive and negative; sensitivity and specificity of Gene-Xpert in diagnosing pulmonary and extrapulmonary TB was found out. The incidence of drug resistance in newly detected TB was also found out.

\section{RESULTS}

The performance of the Gene-Xpert assay for rapid diagnosis of tuberculosis and detection of Rifampicin resistance in pulmonary and extrapulmonary specimens obtained from possible tuberculosis patients was determined. 100 patients who satisfied the inclusion criteria were selected and 62 pulmonary and 38 extrapulmonary specimens of these patients were studied. 49 (41 culture positive and 8 culture negative for M. tuberculosis) of the 100 patients were diagnosed to have tuberculosis. The sensitivity and specificity of Gene-Xpert was found to be $75.5 \%$ and $100 \%$ respectively. In pulmonary specimens, sensitivity and specificity were $79.3 \%$ and $100 \%$ respectively. In extrapulmonary specimens, sensitivity was $70 \%$ and specificity was $100 \%$. In smear positive and negative tuberculosis, sensitivity was $100 \%$ and $62.5 \%$ respevtively. The Gene-Xpert also detected 2 RIF resistant specimens and 35 RIF susceptible specimens. Incidence of RIF resistance in newly detected TB patients was 4.08\% (2/49).

\section{CONCLUSION}

Gene-Xpert sensitivity is higher than AFB smear, both in pulmonary and extrapulmonary specimens. Simultaneous detection of Rifampicin resistance is especially beneficial in patient with MDR and HIV associated Tuberculosis. Early detection of TB and RIF resistance helps to initiate the appropriate therapy, thus improving the quality of TB care. However, Gene-Xpert will not replace conventional microscopy, AFB smear, culture and drug sensitivity. So, this test may be done in the context of a comprehensive algorithm including microscopy, AFB smear, culture and drug sensitivity.

\section{KEY WORDS}

Gene-Xpert, Rifampicin Resistance, RPOB Gene.

HOW TO CITE THIS ARTICLE: Mathew S, Pradhani MM, Philomina B. Role of molecular diagnostic test in diagnosing pulmonary and extrapulmonary tuberculosis. J. Evolution Med. Dent. Sci. 2018;7(23):2777-2781, DOI: 10.14260/jemds/2018/627

\section{BACKGROUND}

$25 \%$ of world's annual incidence of Tuberculosis is contributed by India. Every year about 2 million people develop TB in India and 3 lakhs die of TB. There is a comprehensive national TB program in India, which successfully guides every state for implementation of TB

'Financial or Other Competing Interest': None.

Submission 02-04-2018, Peer Review 22-05-2018,

Acceptance 28-05-2018, Published 04-06-2018.

Corresponding Author:

Dr. Sheela Mathew,

Government Medical College Hospital,

Kozhikode, Kerala, India.

E-mail: shaarongardens@yahoo.com

DOI: $10.14260 /$ jemds $/ 2018 / 627$

\section{(c) $(1)$}

diagnosis and treatment. In spite of it, the incidence is still high and there is a considerable rate of mortality also. The emergence of resistance to drugs used to treat TB has become a major obstacle for effective TB control. WHO estimates approximately $4.5 \mathrm{~L}$ MDR-TB in the world, of which $3 \mathrm{~L}$ cases are pulmonary. ${ }^{1}$ More than $50 \%$ of MDR-TB is contributed by 3 countries- India, China and Russia. $30 \%$ of newly detected TB and $20 \%$ of previously treated cases are MDR-TB. Out of the MDR, 9.7\% is XDR. Presently, XDR is reported from 106 countries and $50 \%$ of MDR and $26 \%$ of XDR only gets successful treatment.

Efficacy of any anti-TB regimen is measured by a successful early outcome indicated by high sputum conversion and high treatment success as well as a successful long-term outcome in the form of low relapse and low 
emergence of drug resistance. The ultimate goal of treatment is cure, breaking chain of transmission and prevention of resistance. In India a set of standards have been set up and recognised as appropriate for specific challenges India is facing while managing a TB patient. 26 standards were setup after a national workshop and labelled as standards of TB care in India. ${ }^{2}$ These standards are used as a benchmark by all providers managing TB patients in India and are set up for detection, management and prevention.

With the intermittent TB regimen in India, $15 \%$ of total notified cases of TB are relapse cases. But in other high burden countries, it is only $7 \%$. The risk of relapse in India is 2.32 times more as compared to other high burden countries. Out of 35 states only 16 states are reporting more than $90 \%$ sputum conversion rate after treatment. In states like Sikkim, Kerala and Meghalaya, the sputum conversion rate falls below $85 \%$. In the context of immune suppression, clinicians frequently encounter extrapulmonary $\mathrm{TB}$ and disseminated TB. Tissue culture may yield positivity in $1 / 3^{\text {rd }}$ of cases. Also, while treating patients, an immune reconstitution syndrome can occur, as for example in a LN TB the nodes may remain persistently enlarged or new LN may appear or even after completion of treatment nodes remain enlarged. Sometimes in case of TB meningitis, the CSF markers may persist or worsen with therapy. So, the treating physician may be in a dilemma whether they are dealing with drug resistant TB or is it a case of TB at all. The ultimate aim is to treat the disease if present, at the same time patients should not be exposed to serious side effects of drugs if disease is not present. Also, there is a public health goal that we must interrupt transmission to others. This highlights the significance of prompt diagnosis.

To diagnose TB we depend on smear microscopy, culture, tissue histopathology and various adjunctive tests like ADA, LDH etc. The sensitivity of smear examination varies from $20 \%-80 \%$. In children, sensitivity is $<50 \%$. Major limitation is when there is low bacillary load, the chance of detection is poor. This happens especially in immune suppressed patients like HIV. Granuloma formation and caseation is minimal, expectorated bacillary load is low leading to delayed or wrong diagnosis and can contribute to high mortality, increases transmission and epidemic continues. Since drug resistance testing is not done routinely MDR TB increases. Hence, what we need is more sensitive, specific and faster tests for diagnosis as well as drug resistance detection.

The standards of TB care in India has clearly mentioned how to screen and test for pulmonary and extrapulmonary TB, both in adults and paediatric population. Accordingly, any person with symptoms and signs suggestive of TB including cough $>2$ weeks, fever $>2$ weeks, significant weight loss, haemoptysis etc. and any abnormality in chest radiograph must be evaluated for TB. Children with persistent fever and/or cough $>2$ weeks, loss of weight/ no weight gain and/or h/o contact with pulmonary $\mathrm{TB}$ cases must be evaluated for TB.3,4

\section{Diagnostic Technology}

1. Microbiological confirmation on sputum- All patients (adults, adolescents and children who are capable of producing sputum) with presumptive pulmonary TB should undergo a quality-assured sputum test for rapid microbiological diagnosis of TB.
2. Chest $\mathrm{x}$-ray as screening tool where available, chest $\mathrm{x}$-ray should be used as a screening tool to increase the sensitivity of the diagnostic algorithm. Serological tests are banned and not recommended for diagnosing tuberculosis. Tuberculin Skin Test (TST) and Interferon Gamma Release Assay (IGRA) are not recommended for the diagnosis of active tuberculosis.

3. Acceptable methods for bacteriologic testing of sputum include sputum smear microscopy, both conventional and fluorescent; culture on solid or liquid media, commercial line probe assay or CB-NAAT. Sputum culture is highly sensitive and specific and utilises either a solid or liquid media. Disadvantage of culture is that it requires weeks to yield results and alone does not help clinicians for early diagnosis. Nucleic acid amplification testing (NAAT) offers enormous potential for accurate rapid diagnosis. PCR was approved in 1995 for AFB smear positive patients and in 1999 enhanced method was approved for smear negative persons. ${ }^{5}$ Sensitivity in smear negative samples were $70 \%$ and smear positive samples were $97 \%$. Specificity was $98 \%$. Some of the limitations were lack of easy availability in hospital labs. It was not easy to perform as it needed manual sample processing and DNA extraction, chance of laboratory contamination and false positivity and presence of inhibiting enzymes in secretions. So, in spite of their theoretical ability to detect even a single mycobacterial cell, NAATs are not sufficiently reliable to replace conventional methods. Key factor for negative PCR is low bacterial load as for smear and culture.

To overcome the limitations of PCR, automated commercial tests are developed, where there is standardised procedure and reagents for sample processing, amplification and detection. Different steps are done in a single sealed tube, minimising chance of contamination. Inhibitors are detected and no chance of false negativity. Gene-Xpert MTB/RIF assay is a novel integrated diagnostic device for the diagnosis of tuberculosis and rapid detection of RIF resistance in clinical specimens. This is an automated cartridge-based test using PCR technology in a self-contained capsule. So contamination is eliminated. Test can be done directly on clinical specimen. Test detects simultaneously Rifampicin resistance and MTB complex.6,7 95\% of Rifampicin resistance is due to rpo-B gene mutation and mutation in this gene is detected by the system. Results are available in 2 hours. Test can be used in smear negative and positive specimen. Also, both pulmonary and extrapulmonary samples can be tested.

\section{Aim of Study \\ Application of newer diagnostic test like Gene-Xpert MTB/RIF assay for diagnosing pulmonary and extrapulmonary tuberculosis in suspected tuberculosis patients.}

\section{Objectives of Study}

- To find out sensitivity and specificity of Gene-Xpert.

- $\quad$ To find out the incidence of drug resistance in newly detected TB with the help of Gene-Xpert. 


\section{MATERIALS AND METHODS Study Design}

This is a prospective observational study done in Government Medical College Hospital, Kozhikode. A total of 100 patients were included in the study. Inclusion criteria was age $>12$ yrs. and clinical or radiological features suggestive of Tuberculosis. Already detected and getting treatment TB cases and drug default cases were excluded.

Those who were selected for the study were evaluated by a detailed clinical history, thorough clinical examination and relevant laboratory investigations. In these suspected tuberculosis patients, diagnosis was confirmed by AFB culture positivity. All samples were subjected for AFB culture, smear microscopy and Gene-Xpert.

Both pulmonary and extrapulmonary samples were received in the Mycobacterium Laboratory. Samples were processed inside biosafety cabinet in negative pressure room. Tissue samples were grinded in sterile pestle mortar. Samples were decontaminated by-

\section{NALC Sodium Hydroxide- Sodium Citrate decontaminating procedure-}

1. Fresh Digestant prepared by mixing $4 \% \mathrm{NaOH}+2.9 \% \mathrm{Na}$ citrate into it, $0.5 \%$.

2. NALC powder per $100 \mathrm{~mL}$ of above solution added, kept for $24 \mathrm{hrs}$.

3. $10 \mathrm{~mL}$ of specimen transferred to $50 \mathrm{~mL}$ plastic centrifuge tube and equal volume of prepared digestant solution were added. Vortex for 5 - 20 seconds and tube inverted and mixed and allowed to stand for $15-20$ minutes.

4. Phosphate buffer solution (PBS) at pH 6.8 were added up to $50 \mathrm{~mL}$ of the tube and mixed by inverting the tube. Centrifuged for 15 minutes at $6000 \mathrm{rpm}$.

5. Supernatant fluid decanted leaving pellet or sediments at the bottom of the tube.

6. Pellet re-suspended with PBS to final volume of $2 \mathrm{~mL}$. Final pH $6.8-7.5$.

\section{Sample Inoculation}

The processed specimen pellet were inoculated into two Loeffler-Jenson (LJ) agar media and incubated at $37^{\circ} \mathrm{C}$. Media examined for any growth and AFB smear taken if growth present. It was considered negative, if no growth for 2 months. Clinical sputum samples (or decontaminated sputum pellets) are treated with a sodium hydroxide and isopropanol containing sample reagent (SR). The SR is added to the sample at a 3: 1 ratio for sputum pellets and a 2: 1 ratio for unprocessed sputum samples and incubated at room temperature for 15 mins. This step is designed to reduce the viability of $\mathrm{M}$. tuberculosis in sputum to reduce biohazard risk. The treated sample is then manually transferred to the cartridge, which is loaded into the Gene-Xpert instrument. Subsequent processing is fully automated.

The cartridge incorporates a syringe drive, a rotary drive and a filter upon which M. tuberculosis bacilli are deposited after being liberated from the clinical material. The test platform employs a sonic horn that inserts into the cartridge base to cause ultrasonic lysis of the bacilli and release of the genetic material. The assay then amplifies a 192 bp segment of the rpo-B gene using a hemi-nested RT-PCR reaction. The assay also contains lyophilized Bacillus globigii spores, which serve as an internal sample processing and PCR control. The B. globigii PCR assay is multiplexed with the M. tuberculosis assay. Mycobacterium tuberculosis is detected by the five overlapping molecular probes (probes A-E) that collectively are complementary to the entire $81 \mathrm{bp}$ rpo-B core region. $\mathrm{M}$. tuberculosis is identified when at least two of the five probes give positive signals with a cycle threshold (CT) of $\leq 38$ cycles and that differ by no more than a pre-specified number of cycles. The B. globigii internal control is positive when the single B. globigii-specific probe produces a CT of $\leq 38$ cycles. The standard user interface indicates the presence or absence of M. tuberculosis and the presence or absence of Rifampicin resistance and a semi-quantitative estimate of the concentration of bacilli as defined by the CT range (high, $<16$; medium, 16-22; low, 22-28; very low, > 28). Assays that are negative for $\mathrm{M}$. tuberculosis and for the B. globigii internal control are reported as invalid assays. When performed on unprocessed sputum samples, the assay can generate results within $2 \mathrm{~h}$ with less than 15 mins of hands-on time. The basis for detection of rifampicin resistance is the difference between the first (early CT) and the last (late CT) M. tuberculosis-specific beacon $(\Delta \mathrm{CT})$.

Patients were diagnosed as microbiologically confirmed case and clinically diagnosed case. Microbiologically confirmed case refers to a presumptive TB patient with biological specimen positive for Acid Fast Bacilli by Xpert or positive for Mycobacterium tuberculosis on culture or on smear. The gold standard for diagnosis is culture positivity.

Clinically diagnosed TB case refers to a presumptive TB patient who is not microbiologically confirmed, but has been diagnosed with active TB on the basis of X-ray abnormalities or clinical signs with a decision to treat the patient with full course anti-TB treatment.

\section{Patient Groups}

Patients were grouped as those with smear and culture positive. Tuberculosis, those with smear negative and culture positive tuberculosis, those who were smear and culture negative for Tuberculosis but who were treated for Tuberculosis on the basis of clinical/ radiological findings.

\section{Analysis}

Whole data was collected in case record format, coded and entered in MS Excel. Data was analysed using SPSS software version 18. Statistical comparison was done using appropriate statistical methods.

\section{RESULTS}

Among 100 cases studied, 36 patients were above the age 50 years and 64 patients were in the age group of 12 - 50 years. 44 were females and 56 were males. Out of 100 patients, 26 were diabetic, 28 were hypertensive, 35 were smokers and 22 were alcoholics. Three patients had history of contact with tuberculosis and four patients out of hundred were HIV positive. Samples included 62 pulmonary and 38 extrapulmonary. Out of 62 pulmonary samples studied, 58 were sputum samples and 4 were bronchoalveolar lavage samples. Out of 38 extrapulmonary specimens studied, 9 were pleural fluid samples, 8 were CSF, 3 were pericardial, 5 were ascitic fluid, 5 were abscess, 3 were joint aspirations and 5 were lymph node aspiration samples. So the majority of extrapulmonary samples studied were pleural fluid and CSF. 
In 100 suspected Tuberculosis patients, 49 were diagnosed to have tuberculosis according to culture positivity, radiological evidence and/or clinically and 51 were non-tuberculosis. 51 of the 100 suspected patients had no bacteriological, clinical, pathological or radiological evidence of tuberculosis on follow-up.

Out of 49 patients who were diagnosed to have Tuberculosis, Gene-Xpert was positive in 37 patients.

\begin{tabular}{|c|c|c|c|}
\hline & Tuberculosis & $\begin{array}{c}\text { Non- } \\
\text { Tuberculosis }\end{array}$ & Total \\
\hline Gene-Xpert & Positive 37 & 0 & 37 \\
\hline & Negative 12 & 51 & 63 \\
\hline & Total 49 & $\mathbf{5 1}$ & $\mathbf{1 0 0}$ \\
\hline \multicolumn{3}{|c|}{ Table 1. Sensitivity and Specificity of Gene-Xpert in } \\
diagnosing Tuberculosis \\
\hline
\end{tabular}

According to the results for 49 tuberculosis patients diagnosed clinically and microbiologically, the sensitivity of the MTB/ RIF test was $75.5 \%$, the specificity was $100 \%$, the positive predictive value was $100 \%$ and negative predictive value was $80.9 \%$.

\begin{tabular}{|c|c|c|c|c|}
\hline & & TB & Non-TB & Total \\
\hline Gene-Xpert & Positive & 23 & 0 & 23 \\
\hline & Negative & 6 & 33 & 39 \\
\hline & Total & $\mathbf{2 9}$ & $\mathbf{3 3}$ & $\mathbf{6 2}$ \\
\hline \multicolumn{3}{|c|}{ Table 2. Sensitivity and Specificity of Gene-Xpert in } \\
diagnosing Pulmonary Tuberculosis \\
\hline
\end{tabular}

Of 62 pulmonary specimens, after obtaining a combination of culture results and clinical data, 29 patients had a diagnosis of pulmonary tuberculosis. Sensitivity is $79 \%$ and specificity is $100 \%$.

\begin{tabular}{|c|c|c|c|c|}
\hline & & TB & Non-TB & Total \\
\hline Gene-Xpert & Positive & 14 & 0 & 14 \\
\hline & Negative & 6 & 18 & 24 \\
\hline & Total & $\mathbf{2 0}$ & $\mathbf{1 8}$ & $\mathbf{3 8}$ \\
\hline
\end{tabular}

Table 3. Sensitivity and Specificity of Gene-Xpert in diagnosing Extrapulmonary Tuberculosis

Of the 38 extrapulmonary specimens examined, 20 patients were diagnosed as extrapulmonary tuberculosis. Sensitivity was $70 \%$ and specificity was $100 \%$.

\section{AFB Smear}

17 patients' AFB smear was positive, out of 49 diagnosed TB cases. Gene-Xpert was positive in all smear positive cases and also positive in 20 out of 32 smear negative cases.

\begin{tabular}{|c|c|c|c|c|}
\hline & & $\begin{array}{c}\text { Smear } \\
\text { Positive TB }\end{array}$ & $\begin{array}{c}\text { Smear } \\
\text { Negative TB }\end{array}$ & Total \\
\hline $\begin{array}{c}\text { Gene- } \\
\text { Xpert }\end{array}$ & Positive & 17 & 20 & 37 \\
\hline & Negative & 0 & 12 & 12 \\
\hline & Total & $\mathbf{1 7}$ & $\mathbf{3 2}$ & $\mathbf{4 9}$ \\
\hline
\end{tabular}

Table 4. Sensitivity of Gene-Xpert in Smear Positive and Smear Negative TB

In smear positive TB sensitivity was $100 \%$ and in smear negative TB sensitivity was $62.5 \%$.

\section{Culture Positive and Negative TB}

Among 49 diagnosed tuberculosis cases, 41 were culture positive and 8 were culture negative. Out of these 41 GeneXpert was positive in 35 patients and also positive in 2 of 8 culture negative cases.

\begin{tabular}{|c|c|c|c|c|}
\hline & $\begin{array}{c}\text { Culture } \\
\text { Positive TB }\end{array}$ & $\begin{array}{c}\text { Culture } \\
\text { Negative TB }\end{array}$ & Total \\
\hline $\begin{array}{c}\text { Gene- } \\
\text { Xpert }\end{array}$ & Positive & 35 & 2 & 37 \\
\hline & Negative & 6 & 6 & 12 \\
\hline & Total & $\mathbf{4 1}$ & $\mathbf{8}$ & $\mathbf{4 9}$ \\
\hline \multicolumn{4}{|c|}{ Table 5. Sensitivity of Gene-Xpert in Culture Positive and } \\
Negative TB
\end{tabular}

Sensitivity in culture positive TB is $85.3 \%$. Sensitivity in culture negative TB is $25 \%$.

\section{Rifampicin Resistance}

The MTB/ RIF assay simultaneously detected 2 RIF-resistant specimens and 35 RIF-susceptible specimens out of 37 positive specimens. So, the incidence of RIF resistance was found to be $4.08 \%(2 / 49)$.

\section{DISCUSSION}

In our study, the performance of the MTB/ RIF assay with pulmonary and extrapulmonary specimens was investigated. The overall sensitivity and specificity was found to be $75.5 \%$ and $100 \%$ respectively. In a study done by Zeka et al, sensitivity and specificity was found to be $70 \%$ and $100 \%$ respectively. 8 The NPV was $80.9 \%$ and PPV was $100 \%$ in our study. Zeka et al showed NPV of $90.6 \%$ and PPV of $100 \%$. The sensitivity of Gene-Xpert in diagnosing pulmonary TB in our study was $79.3 \%$, specificity was $100 \%$. Zeka et al study sensitivity was $82.3 \%$ and specificity was $100 \%$. Gene-Xpert showed a sensitivity of $100 \%$ in smear positive pulmonary TB and a sensitivity of $62.5 \%$ in smear negative pulmonary TB. This result was comparable with a study done by Marlowe et al, where Gene-Xpert had a sensitivity of $98 \%$ in smear positive pulmonary TB and $72 \%$ in smear negative pulmonary TB. ${ }^{9}$ A sensitivity of $70 \%$ and specificity of $100 \%$ of Gene-Xpert in diagnosing extrapulmonary TB was observed in our study. A study done by Tortoli et al (2012) showed a sensitivity of $81.3 \%$ and specificity of $99.8 \%$ respectively. ${ }^{10}$ In another study done in India by Vadwai et al, a sensitivity of $81 \%$ and a specificity of $99.6 \%$ in diagnosing extrapulmonary TB was noted.11 In a study performed by Armand et al, the sensitivities of the test for smear positive and smear negative extrapulmonary specimens have been reported to be $100 \%$ and $37 \%$, respectively. 12 In the present study, the sensitivity of the test was also $100 \%$ for smearpositive extrapulmonary specimens, but in contrast to the findings of Armand et al our results with extrapulmonary specimens revealed $62.5 \%$ sensitivity for smear negative specimens. In all tuberculosis cases, the sensitivity of the MTB/ RIF test for pulmonary specimens was statistically higher than that for extrapulmonary specimens (P- 0.001). It could be because of the high smear-negative rate for nonrespiratory specimens. In our study, Gene-Xpert detected mycobacterium in 23 of 29 pulmonary specimens and 14 of 20 extrapulmonary specimens ( 4 of 9 pleural fluid specimens, 4 of 8 CSF specimens, 1 of 3 pericardial fluid specimens, 3 of 5 
ascitic fluid specimens, 3 of 5 abscess specimens, 2 of 3 joint aspiration specimens, 3 of 5 lymph node aspiration specimens were obtained from 49 patients. The Gene-Xpert test was positive for 35 of 41 culture positive samples and 2 of 8 culture negative samples from clinical tuberculosis cases.

In our study, even though sensitivity of Gene-Xpert was found to be lower than culture, test contributed to the diagnosis of 2 of 49 tuberculosis cases. The sensitivity of microscopy was $48.1 \%$ for culture positive pulmonary specimens and $28.5 \%$ for culture positive extrapulmonary specimens. The Gene-Xpert assay was positive for all of the smear positive specimens, but smear was positive only for 17 of 35 Gene-Xpert positive specimens.

Our study also showed Gene-Xpert sensitivity of $100 \%$ and $62.5 \%$ in smear positive and smear negative $\mathrm{TB}$ respectively. In culture positive and culture negative TB, sensitivity and specificity was $85 \%$ and $25 \%$ respectively.

Our study detected 2 RIF resistant and 35 RIF sensitive samples by Gene-Xpert. Incidence of RIF resistance in newly detected Tuberculosis patients was found to be $4.08 \%$.

\section{CONCLUSION}

Gene-Xpert is useful for rapid detection of TB and RIF resistance. It is a highly sensitive and specific test. Gene-Xpert sensitivity is higher than AFB smear, both in pulmonary and extrapulmonary specimens. Simultaneous detection of Rifampicin resistance is beneficial in patient with MDR and HIV associated tuberculosis. Early detection of TB and RIF resistance helps to initiate the appropriate therapy, thus improving the quality of TB care. However, Gene-Xpert will not replace conventional microscopy, AFB smear, culture and drug sensitivity. So, this test may be done in the context of a comprehensive algorithm including microscopy, AFB smear, culture and drug sensitivity.

\section{REFERENCES}

[1] Nhu NT, Heemskerk D, Thu do DA, et al. Evaluation of GeneXpert MTB/RIF for diagnosis of tuberculous meningitis. J Clin Microbiol 2014;52(1):226-33.

[2] WHO. Standards for TB Care in India. New Delhi: World Health Organisation Country Office for India 2014.
[3] WHO. Global tuberculosis report. 20 $0^{\text {th }}$ edn. Geneva: World Health Organisation 2015.

[4] WHO. Standards of TB care in India. Standards for TB Care in India. New Delhi: World Health Organisation 2016.

[5] Rimek D, Tyagi S, Kappe R. Performance of an IS6110based PCR assay and the COBAS AMPLICOR MTB PCR system for detection of Mycobacterium tuberculosis complex DNA in human lymph node samples. J Clin Microbiol 2002;40(8):3089-92.

[6] Helb D, Jones M, Story E, et al. Rapid detection of Mycobacterium tuberculosis and rifampin resistance by use of on-demand, near-patient technology. J Clin Microbiol 2010;48(1):229-37.

[7] Blakemore R, Story E, Helb D, et al. Evaluation of the analytical performance of the Xpert MTB/RIF assay. J Clin Microbiol 2010;48(7):2495-501.

[8] Zeka AN, Tasbakan S, Cavusoglu C. Evaluation of the GeneXpert MTB/RIF assay for rapid diagnosis of tuberculosis and detection of rifampin resistance in pulmonary and extrapulmonary specimens. J Clin Microbiol 2011;49(12):4138-41.

[9] Marlowe EM, Novak-Weekley SM, Cumpio J, et al. Evaluation of the Cepheid Xpert MTB/RIF assay for direct detection of Mycobacterium tuberculosis complex in respiratory specimens. J Clin Microbiol 2011;49(4):1621-3.

[10] Tortoli E, Russo C, Piersimoni C, et al. Clinical validation of Xpert MTB/RIF for the diagnosis of extrapulmonary tuberculosis. Eur Respir J 2012;40(2):442-7.

[11] Vadwai V, Boehme C, Nabeta P, et al. Xpert MTB/RIF: a new pillar in diagnosis of extrapulmonary tuberculosis? J Clin Microbiol 2011;49(7):2540-5.

[12] Armand S, Vanhuls P, Delcroix G, et al. Comparison of the Xpert MTB/RIF test with an IS6110-TaqMan realtime PCR assay for direct detection of Mycobacterium tuberculosis in respiratory and nonrespiratory specimens. J Clin Microbiol 2011;49(5):1772-6. 